Supporting Information for

\title{
Synthesis of Bridgehead Hydroxy Bicyclo[2.2.2]octane derivatives
}

Viveca Thornqvist, Sophie Manner, Magnus Wingstrand and Torbjörn Frejd*

Division of Organic Chemistry, Kemicentrum, Lund University, P.O. Box 124, SE-221 00 Lund, Sweden.

Torbjorn.Frejd@organic.lu.se

$\underline{\text { Table of Contents: }}$

General and detailed Experimental Methods S3-S13

Table 1: results from hydroboration-oxidation attempts. S14-S15

${ }^{1} \mathrm{H}$ NMR spectrum of 9 S16

${ }^{13} \mathrm{C}$ NMR spectrum of 9 S17

${ }^{1} \mathrm{H}$ NMR spectrum of $\mathbf{1 0}$ S18

${ }^{13} \mathrm{C}$ NMR spectrum of $\mathbf{1 0}$ S19

${ }^{1} \mathrm{H}$ NMR spectrum of $\mathbf{1 4}$ S20

${ }^{13} \mathrm{C}$ NMR spectrum of $\mathbf{1 4}$ S21

${ }^{1} \mathrm{H}$ NMR spectrum of $\mathbf{1 6}$ S22

${ }^{13} \mathrm{C}$ NMR spectrum of $\mathbf{1 6}$ . $\mathrm{S} 23$ 


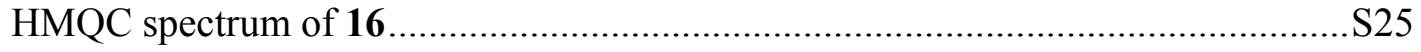

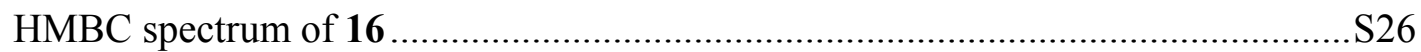

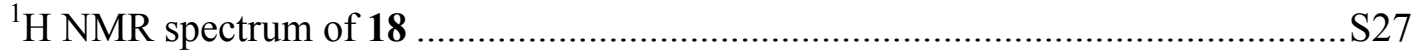

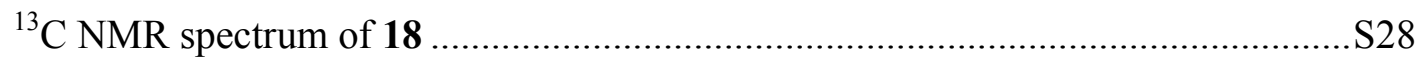

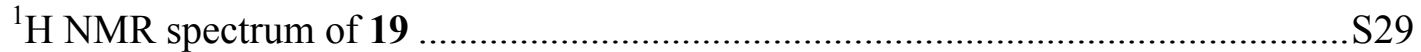

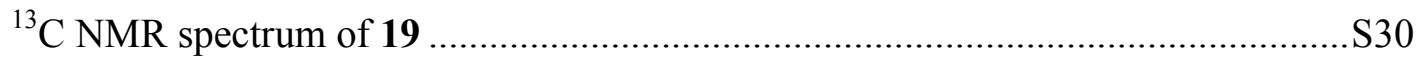

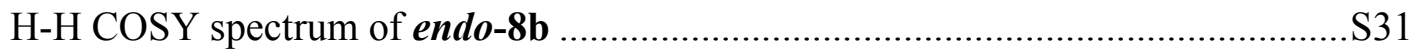

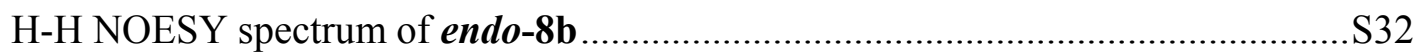

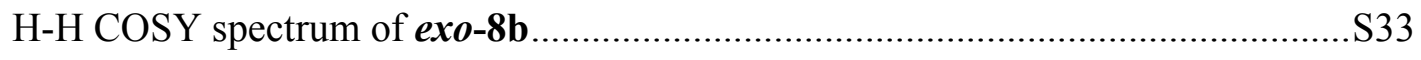

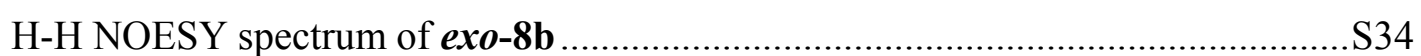




\section{Experimental Methods}

General: Materials were obtained from commercial suppliers and were used without further purification unless otherwise stated. The indium powder quality used was -100 mesh, 99.99\%. Silver(I)oxide was freshly prepared through addition of an aqueous sodium hydroxide solution (1M) to an aqueous silver nitrate solution $(10 \% \mathrm{w} / \mathrm{w})$, collected by filtration, dried over $\mathrm{P}_{2} \mathrm{O}_{5}$, and stored in a brown glass jar. Methanol was dried by standing over $3 \AA$ molecular sieves for $48 \mathrm{~h}$. Other solvents were dried and distilled according to standard procedures. All moisture and airsensitive reactions were carried out under an atmosphere of dry argon using oven-dried glassware. GC chromatographic analyses were performed on a gas chromatograph equipped with a capillary column $(30 \mathrm{~m}, 0.25 \mathrm{~mm}$ i.d., $0.25 \mu \mathrm{m}$ stationary phase). The NMR spectra were recorded at 300 $\mathrm{MHz}\left({ }^{1} \mathrm{H}\right)$ and at $75 \mathrm{MHz}\left({ }^{13} \mathrm{C}\right)$, at $400 \mathrm{MHz}\left({ }^{1} \mathrm{H}\right)$ and at $100 \mathrm{MHz}\left({ }^{13} \mathrm{C}\right)$ or at $500 \mathrm{MHz}\left({ }^{1} \mathrm{H}\right)$ and at $125 \mathrm{MHz}\left({ }^{13} \mathrm{C}\right)$ using $\mathrm{CDCl}_{3}\left(\mathrm{CHCl}_{3} \delta 7.26\left({ }^{1} \mathrm{H}\right)\right.$ and $\left.77.0\left({ }^{13} \mathrm{C}\right)\right)$ or benzene- $d_{6}\left(\mathrm{C}_{6} \mathrm{H}_{6} \delta 7.16\left({ }^{1} \mathrm{H}\right)\right.$ and $\left.128.0\left({ }^{13} \mathrm{C}\right)\right)$ as solvents. NOESY spectra were acquired using the TPPI method and $2048 \times$ 512 complex data points on a spectral width of $3531 \mathrm{~Hz}$. The mixing time used was 1 second and the relaxation delay was 1.5 second for endo-5b and 2 seconds for exo-5b. The melting points were not corrected. Chromatographic separations were performed on normal phase silica gel 60 (0.035-0.070 mm). TLC was performed on precoated TLC glass plates coated with silica gel $60 \mathrm{~F}_{254}, 0.25 \mathrm{~mm}$. After elution, the TLC-plates were sprayed with a solution of p-methoxybenzaldehyde $(26 \mathrm{~mL})$, glacial acetic acid $(11 \mathrm{~mL})$, concentrated sulphuric acid $(35 \mathrm{~mL})$ and $95 \%$ ethanol $(960 \mathrm{~mL})$ or a solution of $\mathrm{KMnO}_{4},(10 \mathrm{~g}), \mathrm{K}_{2} \mathrm{CO}_{3}(50 \mathrm{~g}), \mathrm{NaOH}(20 \mathrm{~mL}, 5 \%)$ and $\mathrm{H}_{2} \mathrm{O}(900 \mathrm{~mL})$ and the compounds were visualized upon heating.

( \pm )-7-Allyl-1,4-dioxa-spiro[4.5]decan-7-ol (5a). Synthesis of 1,4-dioxaspiro[4,5]decane-7-one 4 was performed according to the protocol of

Takagi et al. ${ }^{1}$ The resulting product, a colourless liquid, was obtained as a mixture of $\mathbf{4}$ and the corresponding diketal 1,4,8,11-

\footnotetext{
${ }^{1}$ Takagi, H.; Hayashi, T.; Mizutani, T.; Masuda, H.; Ogoshi, H.; Journal of the Chemical Society, Perkin Transactions 1: Organic and BioOrganic Chemistry 1999, 1885-92.
} 
tetraoxadispiro[4.1.4.3]tetradecane in a 77:23 ratio respectively, according to GC analysis. The mixture was used without further purification in the next step.

At $0{ }^{\circ} \mathrm{C}$ indium powder $(4.41 \mathrm{~g}, 38.4 \mathrm{mmol})$ was added in one portion to a solution containing 4 (3.0 g, $\left.19.2 \mathrm{mmol}\right)$ and allyl bromide (6.97 g, $57.6 \mathrm{mmol})$ in $\mathrm{MeOH}(40 \mathrm{~mL})$. The reaction mixture was stirred for 40 minutes at $0{ }^{\circ} \mathrm{C}$ where after it was filtered through a pad of celite. After concentration at reduced pressure the residue was purified by column chromatography $\left(\mathrm{SiO}_{2}\right.$, heptane:EtOAc 8:2) to give 5a (3.42 g, $90 \%$ based on the amount of 10) as a colourless liquid; IR $(\mathrm{NaCl}) 3526,3074,2885,1639 \mathrm{~cm}^{-1}$; ${ }^{1} \mathrm{H}$ NMR $\left(400 \mathrm{MHz}\right.$, benzene- $\left.d_{6}\right) \delta 6.12(\mathrm{ddt}, J=17.1,10.1$, $7.3 \mathrm{~Hz}, 1 \mathrm{H}) 5.11-5.03(\mathrm{~m}, 2 \mathrm{H}) 3.99(\mathrm{~s}, 1 \mathrm{H}) 3.40-3.27(\mathrm{~m}, 4 \mathrm{H}) 2.32\left(\mathrm{br} \mathrm{dd}_{\mathrm{AB}}, J=7.0 \mathrm{~Hz}, J_{\mathrm{AB}}=13.7 \mathrm{~Hz}, 1 \mathrm{H}\right) 2.21\left(\right.$ br dd $\mathrm{AB}, J=7.5 \mathrm{~Hz}, J_{\mathrm{AB}}=13.0$ $\mathrm{Hz}, 1 \mathrm{H}) 1.89(\mathrm{tq}, J=3.7,13.5 \mathrm{~Hz}, 1 \mathrm{H}) 1.76\left(\mathrm{td}_{\mathrm{AB}}, J=2.5 \mathrm{~Hz}, J_{\mathrm{AB}}=13.7 \mathrm{~Hz}, 1 \mathrm{H}\right) 1.65-1.60(\mathrm{~m}, 2 \mathrm{H}) 1.50\left(\mathrm{~d}_{\mathrm{AB}}, J_{\mathrm{AB}}=13.7 \mathrm{~Hz}, 1 \mathrm{H}\right) 1.47-1.42(\mathrm{~m}$, 1H) $1.33(\mathrm{dt}, J=4.4,13.2 \mathrm{~Hz}, 1 \mathrm{H}) 1.04(\mathrm{dt}, J=4.2,13.3 \mathrm{~Hz}, 1 \mathrm{H}) ;{ }^{13} \mathrm{C}$ NMR $\left(100 \mathrm{MHz}\right.$, benzene- $\left.d_{6}\right) \delta 135.0,117.3,109.9,72.2,64.3,63.9,48.0$, 43.9, 36.7, 35.0, 19.3. HRMS (FAB+): calcd. for $\mathrm{C}_{11} \mathrm{H}_{19} \mathrm{O}_{3}(\mathrm{M}+\mathrm{H})$ : 199.1334. Found: 199.1337. Anal. calcd. for $\mathrm{C}_{11} \mathrm{H}_{18} \mathrm{O}_{3}$ : $\mathrm{C}, 66.64 ; \mathrm{H}, 9.15$. Found: $\mathrm{C}, 66.51 ; \mathrm{H}, 9.23$. Several other solvents were tested in this reaction $\left(\mathrm{MeOH}: \mathrm{H}_{2} \mathrm{O}\right.$ 9:1, EtOH, EtOH: $\mathrm{H}_{2} \mathrm{O}$ 9:1, DMF, THF, THF: $\mathrm{H}_{2} \mathrm{O}$ and $\mathrm{H}_{2} \mathrm{O}$ ) but gave worse results regarding yield.

( \pm -3-((1,3-Dioxolan-2-yl)methyl)cyclohex-2-enone (10). Attempted synthesis of 8a, starting from 5a. Ozone was bubbled through a solution of $5 \mathbf{a}(398 \mathrm{mg}, 2.0 \mathrm{mmol})$ in $\mathrm{CH}_{2} \mathrm{Cl}_{2}(10 \mathrm{~mL})$ at $-78{ }^{\circ} \mathrm{C}$ until it turned light blue (ca. $30 \mathrm{~min}$.). Excess ozone was then driven off by flushing with argon, where after TEA $(1.5 \mathrm{~mL})$ was added, and the reaction mixture was allowed to reach room temperature. After dilution with EtOAc (40 mL), the organic phase was washed with brine $(20 \mathrm{~mL})$ and dried $\left(\mathrm{MgSO}_{4}\right)$. After concentration at reduced pressure the residue was purified by column chromatography $\left(\mathrm{SiO}_{2}\right.$, heptane:EtOAc 7:3 + 10\% EtOH) to give 9 as a colourless oil $(218 \mathrm{mg}, 55 \%) . \mathrm{IR}(\mathrm{NaCl}) 3447,2852,2746,1700 \mathrm{~cm}^{-1} ;{ }^{1} \mathrm{H}$ NMR $\left(300 \mathrm{MHz}, \mathrm{CDCl}_{3}\right) \delta 9.89(\mathrm{t}, J=3.0 \mathrm{~Hz}, 1 \mathrm{H}) 4.17(\mathrm{br} \mathrm{s}, 1 \mathrm{H}) 3.97-3.94(\mathrm{~m}, 4 \mathrm{H}) 2.51\left(\mathrm{dd}_{\mathrm{AB}}, J=2.6 \mathrm{~Hz}, J_{\mathrm{AB}}=15.0 \mathrm{~Hz}, 1 \mathrm{H}\right) 2.48\left(\mathrm{dd} \mathrm{AB}_{1}, J=3.0\right.$ 
$\left.\mathrm{Hz}, J_{\mathrm{AB}}=15.1 \mathrm{~Hz}, 1 \mathrm{H}\right) 1.86-1.68(\mathrm{~m}, 6 \mathrm{H}) 1.53-1.31(\mathrm{~m}, 2 \mathrm{H}) ;{ }^{13} \mathrm{C} \mathrm{NMR}\left(75 \mathrm{MHz}, \mathrm{CDCl}_{3}\right) \delta 202.7,109.0,72.0,64.5,64.1,55.1,44.0,37.0,34.2$, 18.7 .

A solution of 9 in acetone: $2 \mathrm{M} \mathrm{HCl}(100: 1$ or $200: 1,30 \mathrm{~mL})$, was stirred at rt for $24 \mathrm{~h}$, and then heated at reflux for $5 \mathrm{~h}$. After cooling and neutralisation (aqueous $1 \mathrm{M} \mathrm{NaOH})$ the mixture was concentrated at reduced pressure, dissolved in water and extracted with $\mathrm{CH}_{2} \mathrm{Cl}_{2}(9 \times 25 \mathrm{~mL}$ ). The organic phase was then washed with water and brine and dried $\left(\mathrm{Na}_{2} \mathrm{SO}_{4}\right)$. After concentration at reduced pressure the residue was purified by column chromatography $\left(\mathrm{SiO}_{2}\right.$, heptane:EtOAc 7:3) to give $\mathbf{1 0}$ as a colourless oil (32 mg, 35\%). IR ( $\left.\mathrm{NaCl}\right) 2888,1670 \mathrm{~cm}^{-1}$; ${ }^{1} \mathrm{H} \mathrm{NMR}(300 \mathrm{MHz}$, benzene- $\left.d_{6}\right) \delta 6.06(\mathrm{~s}, 1 \mathrm{H}) 4.70(\mathrm{t}, J=4.9 \mathrm{~Hz}, 1 \mathrm{H}) 3.49-3.18(\mathrm{~m}, 4 \mathrm{H}) 2.24(\mathrm{~d}, J=4.9 \mathrm{~Hz}, 2 \mathrm{H}) 2.08(\mathrm{br} \mathrm{t}, J=6.2 \mathrm{~Hz}, 2 \mathrm{H}) 1.82(\mathrm{br} \mathrm{t}, J=5.9 \mathrm{~Hz}, 2 \mathrm{H})$ $1.43(\mathrm{p}, J=6.3 \mathrm{~Hz}, 2 \mathrm{H}) ;{ }^{13} \mathrm{C}$ NMR $\left(75 \mathrm{MHz}\right.$, benzene- $\left.d_{6}\right) \delta 197.3,158.6,128.7,102.9,64.7,42.6,37.4,30.2,22.7$. HRMS (FAB+) calcd. for $\mathrm{C}_{10} \mathrm{H}_{15} \mathrm{O}_{3}(\mathrm{M}+\mathrm{H}):$ 183.1021. Found: 183.1042 .

( \pm )-(7-Allyl-1,4-dioxa-spiro[4.5]dec-7-yloxy)-tert-butyldimethyl-silane (5b). At $0{ }^{\circ} \mathrm{C}$ TBDMSOTf (6.32 g, 23.9 mmol) was added drop wise to a solution containing $\mathbf{5 a}(2.5 \mathrm{~g}, 12.6 \mathrm{mmol})$ and 2,6-lutidine $(4.19 \mathrm{~g}, 39.1 \mathrm{mmol})$ in $\mathrm{CH}_{2} \mathrm{Cl}_{2}(50 \mathrm{~mL})$. The mixture was stirred for $40 \mathrm{minutes}$ at 0 ${ }^{\circ} \mathrm{C}$ and was then diluted with $\mathrm{CH}_{2} \mathrm{Cl}_{2}(50 \mathrm{~mL})$. The organic phase was washed with $\mathrm{H}_{2} \mathrm{O}(2 \times 70 \mathrm{~mL})$, aqueous $10 \% \mathrm{HCl}(2 \times 70 \mathrm{~mL})$, saturated aqueous $\mathrm{NaHCO}_{3}(2 \times 70 \mathrm{~mL})$, brine $(70 \mathrm{~mL})$ and dried $\left(\mathrm{MgSO}_{4}\right)$. After concentration at reduced pressure the residue was purified by column chromatography $\left(\mathrm{SiO}_{2}\right.$, Heptane:EtOAc 95:5) to give 5b $(3.69 \mathrm{~g}, 95 \%)$ as a pale yellow liquid; IR (NaCl) $3074,2855,1639 \mathrm{~cm}^{-1} ;{ }^{1} \mathrm{H} \mathrm{NMR}(400$ $\mathrm{MHz}$, benzene- $\left.d_{6}\right) \delta$ 6.15-6.05 (m, 1H) 5.18-5.12 (m, 2H) 3.52-3.46 (m, 4H) 2.59-2.55 (m, 2H) $2.08\left(\operatorname{td}_{\mathrm{AB}}, J=2.0 \mathrm{~Hz}, J_{\mathrm{AB}}=13.3 \mathrm{~Hz}, 1 \mathrm{H}\right) 1.83$ $\left(\mathrm{d}_{\mathrm{AB}}, J_{\mathrm{AB}}=13.3 \mathrm{~Hz}, 1 \mathrm{H}\right) 1.72-1.63(\mathrm{~m}, 2 \mathrm{H}) 1.54-1.47(\mathrm{~m}, 3 \mathrm{H}) 1.41-1.34(\mathrm{~m}, 1 \mathrm{H}) 1.01(\mathrm{~s}, 9 \mathrm{H}) 0.12(\mathrm{~s}, 6 \mathrm{H})$; ${ }^{13} \mathrm{C}$ NMR $\left(100 \mathrm{MHz}\right.$, benzene- $\left.d_{6}\right) 135.8$, 117.4, 109.4, 76.6, 64.5, 63.9, 46.4, 44.7, 38.4, 35.3, 26.2, 20.2, 18.5, -1.6; HRMS (FAB+) calcd. for $\mathrm{C}_{17} \mathrm{H}_{31} \mathrm{O}_{3} \mathrm{Si}(\mathrm{M}-\mathrm{H})$ : 311.2043. Found: 311.2042. Anal. calcd. for $\mathrm{C}_{17} \mathrm{H}_{32} \mathrm{O}_{3} \mathrm{Si}: \mathrm{C}, 65.33 ; \mathrm{H}, 10.32$. Found: $\mathrm{C}, 65.21 ; \mathrm{H}, 10.27$. 
( \pm )-7-Allyl-7-benzyloxy-1,4-dioxa-spiro[4.5]decane (5c). ${ }^{2}$ A solution of $\mathbf{5 a}(500 \mathrm{mg}, 2.5 \mathrm{mmol})$, benzyl bromide $(890 \mu \mathrm{L}, 7.5 \mathrm{mmol})$ and silver(I)oxide $(1.74 \mathrm{~g}, 7.5 \mathrm{mmol})$ in EtOAc $(17 \mathrm{~mL})$ was stirred at $\mathrm{rt}$ for $48 \mathrm{~h}$. The reaction mixture was filtered through a pad of celite, where after the solvent was removed at reduced pressure. The residue was purified by column chromatography $\left(\mathrm{SiO}_{2}\right.$, pentane:ether 9:1) to give $\mathbf{5} \mathbf{c}$ as a pale yellow oil (266 mg, 37\%). IR (NaCl) 2945, 1639, $1452 \mathrm{~cm}^{-1}$; ${ }^{1} \mathrm{H}$ NMR (400 MHz, benzene-d $) \delta 7.46$ (br d, $\left.J=7.4 \mathrm{~Hz}, 2 \mathrm{H}\right) 7.24$ (br t, $J=7.6 \mathrm{~Hz}$, 2H) 7.15-7.09 (m, 1H) 6.03-5.93 (m, 1H) 5.13-5.08 (m, 2H) $4.41\left(\mathrm{~d}_{\mathrm{AB}}, J_{\mathrm{AB}}=11.0 \mathrm{~Hz}, 1 \mathrm{H}\right) 4.28\left(\mathrm{~d}_{\mathrm{AB}}, J_{\mathrm{AB}}=11.0 \mathrm{~Hz}, 1 \mathrm{H}\right) 3.52-3.47(\mathrm{~m}, 4 \mathrm{H}) 2.52$ $\left(\right.$ br tdd $\left.\operatorname{tdB}_{\mathrm{AB}}, J=1.1,6.7 \mathrm{~Hz}, J_{\mathrm{AB}}=14.8 \mathrm{~Hz}, 1 \mathrm{H}\right) 2.37\left(\right.$ br tddd $\left.\operatorname{taB}, J=1.1,7.5 \mathrm{~Hz}, J_{\mathrm{AB}}=14.8 \mathrm{~Hz}, 1 \mathrm{H}\right) 2.01\left(\right.$ br d $\left.\mathrm{AB}_{\mathrm{AB}}, J_{\mathrm{AB}}=13.9 \mathrm{~Hz}, 1 \mathrm{H}\right) 1.84\left(\right.$ br d $\mathrm{d}_{\mathrm{AB}}, J_{\mathrm{AB}}$ $=13.9 \mathrm{~Hz}, 1 \mathrm{H}) 1.79-1.75(\mathrm{~m}, 1 \mathrm{H}) 1.62-1.54(\mathrm{~m}, 3 \mathrm{H}) 1.50-1.43(\mathrm{~m}, 2 \mathrm{H}) ;{ }^{13} \mathrm{C}$ NMR $\left(100 \mathrm{MHz}\right.$, benzene- $\left.d_{6}\right) \delta 140.4,134.9,128.4,127.8,127.2$, 117.4, 109.5, 77.0, 64.2, 64.1, 63.0, 42.1, 40.6, 35.4, 34.6, 19.7; HRMS (FAB+) calcd. for $\mathrm{C}_{18} \mathrm{H}_{25} \mathrm{O}_{3}(\mathrm{M}+\mathrm{H})$ : 289.1803. Found: 289.1803. Anal. calcd. for $\mathrm{C}_{18} \mathrm{H}_{24} \mathrm{O}_{3}$ : C, 74.97; H, 8.39. Found: C, 75.12; H, 8.40.

General procedure for the ketal deprotection of 5a-c to give 6a-c. Standard conditions. A solution of 5 (1 mmol) in acetone (14 mL) was cooled to $-15{ }^{\circ} \mathrm{C}(\mathbf{5 a})$ or $0{ }^{\circ} \mathrm{C}(\mathbf{5 b}$ and $\mathbf{5 c}) \cdot \mathrm{PdCl}_{2}\left(\mathrm{CH}_{3} \mathrm{CN}\right)_{2}(5 \mathrm{~mol} \%)$ was then added and the reaction flask was covered with aluminium foil to protect the catalyst from light. The progression of the reaction was followed by TLC. After completion, the dark orange reaction mixture was diluted with EtOAc and was washed with ice cold brine until the organic phase became pale yellow (removal of catalyst). The organic phase was dried $\left(\mathrm{Na}_{2} \mathrm{SO}_{4}\right)$, the solvent removed at reduced pressure and the residue purified by column chromatography.

( \pm )-3-Allyl-3-hydroxy-cyclohexanone (6a): The reaction was completed after $4 \mathrm{~h}$ and $\mathbf{6 a}$ was obtained as a colourless oil in a yield of 85-90\%. $\mathrm{R}_{\mathrm{f}}=0.14\left(\mathrm{SiO}_{2}\right.$, heptane:EtOAc 7:3); IR (NaCl) 3431, 3074, 2878, $1693 \mathrm{~cm}^{-1} ;{ }^{1} \mathrm{H}$ NMR (400 MHz, benzene- $\left.d_{6}\right) \delta 5.68-5.58(\mathrm{~m}, 1 \mathrm{H}) 5.01-4.89(\mathrm{~m}$,

${ }^{2}$ Other methods tested for the benzyl protection: (a) Kanai, K., Sakamoto, I., Ogawa, S., Suani, T.; Bull. Chem. Soc. Jpn. 1999, 1529-31. (b) Berry, J. M., Hall, L. D.; Carbohydrate Research 1976, 307-10. (c) Eckenberg, P., Groth, U., Huhn, T., Richter, N., Schmeck, C.; Tetrahedron 1993, 1619-1624. 
2H) $2.24-2.16(\mathrm{~m}, 2 \mathrm{H})$ 1.97-1.88 (m, 4H) 1.81-1.75 (m, 2H) 1.40-1.35 (m, 2H) 1.18-1.11 (m, 1H); ${ }^{13} \mathrm{C}$ NMR $\left(100 \mathrm{MHz}\right.$, benzene- $\left.d_{6}\right) \delta 208.8$, 133.2, 118.9, 74.9, 52.9, 47.5, 40.7, 35.2, 21.1; HRMS (FAB+) calcd. for $\mathrm{C}_{9} \mathrm{H}_{15} \mathrm{O}_{2}(\mathrm{M}+\mathrm{H})$ : 155.1072. Found: 155.1071. Anal. calcd. for $\mathrm{C}_{9} \mathrm{H}_{14} \mathrm{O}_{2}$ : C, 70.10; H, 9.15. Found: C, 69.95; H, 9.07.

( \pm )-3-Allyl-3-(tert-butyl-dimethyl-silanyloxy)-cyclohexanone (6b): The reaction was completed after $1.5 \mathrm{~h}$ and $\mathbf{6 b}$ was obtained as a colourless oil in a yield of $90 \% . \mathrm{R}_{\mathrm{f}}=0.17\left(\mathrm{SiO}_{2}\right.$, heptane:EtOAc 9:1); IR $(\mathrm{NaCl}) 3078,2858,1717 \mathrm{~cm}^{-1} ;{ }^{1} \mathrm{H} \mathrm{NMR}\left(400 \mathrm{MHz}\right.$, benzene- $\left.d_{6}\right) \delta 5.70-$ $5.59(\mathrm{~m}, 1 \mathrm{H}) 5.01-4.89(\mathrm{~m}, 2 \mathrm{H}) 2.28\left(\operatorname{td}_{\mathrm{AB}}, J=2.0 \mathrm{~Hz}, J_{\mathrm{AB}}=13.8 \mathrm{~Hz}, 1 \mathrm{H}\right) 2.14-2.07(\mathrm{~m}, 2 \mathrm{H}) 2.09\left(\operatorname{br} \mathrm{d}_{\mathrm{AB}}, J_{\mathrm{AB}}=14.2 \mathrm{~Hz}, 1 \mathrm{H}\right) 1.88-1.73(\mathrm{~m}, 2 \mathrm{H})$ $1.43-1.25(\mathrm{~m}, 4 \mathrm{H}) 0.93(\mathrm{~s}, 9 \mathrm{H}) 0.11(\mathrm{~s}, 3 \mathrm{H}) 0.03(\mathrm{~s}, 3 \mathrm{H}) ;{ }^{13} \mathrm{C}$ NMR $\left(100 \mathrm{MHz}\right.$, benzene- $\left.d_{6}\right) \delta$ 207.3, 133.9, 118.4, 78.9, 53.3, 47.5, 40.4, 36.1, 26.1, 20.9, 18.6, -1.8, -1.9; HRMS (FAB+) calcd. for $\mathrm{C}_{15} \mathrm{H}_{29} \mathrm{O}_{2} \mathrm{Si}(\mathrm{M}+\mathrm{H})$ : 269.1937. Found: 269.1937. Anal. calcd. for $\mathrm{C}_{15} \mathrm{H}_{28} \mathrm{O}_{2} \mathrm{Si}: \mathrm{C}, 67.11$; $\mathrm{H}, 10.51$. Found: C, 66.97; H, 10.44.

( \pm )-3-Allyl-3-benzyloxy-cyclohexanone (6c): The reaction was completed after $4 \mathrm{~h}$ and $\mathbf{6 c}$ was obtained as a colourless oil in a yield of $86 \%$. $\mathrm{R}_{\mathrm{f}}$ $=0.22\left(\mathrm{SiO}_{2}\right.$, pentane:ether 8:2); IR (NaCl) 3065, 2943, 1713, $1452 \mathrm{~cm}^{-1} ;{ }^{1} \mathrm{H}$ NMR $(400 \mathrm{MHz}$, benzene-d $) \delta 7.29(\mathrm{br} \mathrm{d}, J=7.1 \mathrm{~Hz}, 2 \mathrm{H}), 7.18-7.06$ $(\mathrm{m}, 3 \mathrm{H}) 5.70-5.59(\mathrm{~m}, 1 \mathrm{H}) 5.01-4.89(\mathrm{~m}, 2 \mathrm{H}) 4.21\left(\mathrm{~d}_{\mathrm{AB}}, J_{\mathrm{AB}}=10.9 \mathrm{~Hz}, 1 \mathrm{H}\right) 4.07\left(\mathrm{~d}_{\mathrm{AB}}, J_{\mathrm{AB}}=10.9 \mathrm{~Hz}, 1 \mathrm{H}\right) 2.54\left(\mathrm{td}_{\mathrm{AB}}, J=2.2 \mathrm{~Hz}, J_{\mathrm{AB}}=14.1 \mathrm{~Hz}\right.$, $1 \mathrm{H}) 2.19-2.17(\mathrm{~m}, 1 \mathrm{H}) 2.04\left(\mathrm{br} \mathrm{dd} \mathrm{AB}, J=7.0 \mathrm{~Hz}, J_{\mathrm{AB}}=14.4 \mathrm{~Hz}, 1 \mathrm{H}\right) 1.97\left(\mathrm{br} \mathrm{dd}_{\mathrm{AB}}, J=7.4 \mathrm{~Hz}, J_{\mathrm{AB}}=14.4 \mathrm{~Hz}, 1 \mathrm{H}\right) 1.89\left(\mathrm{~d}_{\mathrm{AB}}, J_{\mathrm{AB}}=14.1 \mathrm{~Hz}, 1 \mathrm{H}\right)$ 1.79-1.75 (m, 2H) 1.64-1.60 (m, 1H) 1.32-1.29 (m, 1H) 1.13-1.09 (m, 1H); ${ }^{13} \mathrm{C}$ NMR (100 MHz, benzene- $\left.d_{6}\right) \delta 207.2,139.2,133.3,128.5,127.6$, 127.5, 118.1, 79.6, 63.1, 49.7, 41.7, 40.5, 33.0, 20.8; HRMS (FAB+) calcd. for $\mathrm{C}_{16} \mathrm{H}_{21} \mathrm{O}_{2}(\mathrm{M}+\mathrm{H})$ : 245.1541. Found: 245.1540. Anal. calcd. for $\mathrm{C}_{16} \mathrm{H}_{20} \mathrm{O}_{2}$ : C, 78.65; H, 8.25. Found: C, 78.55; H, 8.27.

( \pm )-endo/exo-2,4-Dihydroxybicyclo[2.2.2]octan-6-one (8a). Ozone was bubbled through a solution of $\mathbf{6 a}\left(60 \mathrm{mg}, 38 \mathrm{mmol}^{2}\right.$ in $\mathrm{CH}_{2} \mathrm{Cl}_{2}(8.7$ $\mathrm{mL})$ at $-78^{\circ} \mathrm{C}$ until it turned light blue (ca. 10 min.). Excess ozone was then driven off by flushing with argon, where after DMS (148 $\left.\mu \mathrm{L}\right)$ was added and then the reaction mixture was allowed to reach room temperature. The solvent was removed at reduced pressure and to the resulting 
yellow oil was added heptane $(4.7 \mathrm{~mL})$, toluene $(2.4 \mathrm{~mL})$ and TEA $(1.7 \mathrm{~mL})$. Under intense stirring $\mathrm{SiO}_{2}\left(450 \mathrm{~m}^{2} / \mathrm{g}\right)(1.7 \mathrm{~g})$ was added and after a couple of minutes the colour had turned deep red. After completion of reaction (TLC) the silica gel was filtered off and washed with EtOAc. The filtrate was collected, the solvent removed at reduced pressure and the residue purified by column chromatography $\left(\mathrm{SiO}_{2}\right.$, toluene:EtOAc $\left.1: 9\right)$ to give a barely separable mixture of endo-8a and exo-8a $(27 \mathrm{mg}, 45 \%)$ as a white solid.

endo-8a: $\mathrm{mp}$ decomposes; IR (KBr) 3402, 2951, $1717 \mathrm{~cm}^{-1} ;{ }^{1} \mathrm{H}$ NMR $\left(400 \mathrm{MHz}\right.$, pyridine- $\left.d_{5}\right) \delta 4.49-4.45(\mathrm{~m}, 1 \mathrm{H}) 3.00\left(\mathrm{dd}_{\mathrm{AB}}, J=3.0, J_{\mathrm{AB}}=17.9\right.$ $\mathrm{Hz}, 1 \mathrm{H}) 2.78-2.72(\mathrm{~m}, 1 \mathrm{H}) 2.74\left(\mathrm{dd}_{\mathrm{AB}}, J=3.3, J_{\mathrm{AB}}=17.7 \mathrm{~Hz}, 1 \mathrm{H}\right) 2.63-2.59(\mathrm{~m}, 1 \mathrm{H}) 2.29-2.25(\mathrm{~m}, 1 \mathrm{H}) 1.89-1.83(\mathrm{~m}, 2 \mathrm{H}) 1.74-1.69(\mathrm{~m}, 2 \mathrm{H}) ;{ }^{13} \mathrm{C}$ NMR $\left(100 \mathrm{MHz}\right.$, pyridine- $\left.d_{5}\right) \delta 212.9,69.8,68.3,53.3,51.7,46.2,33.2,19.7$; HRMS (FAB + ) calcd. for $\mathrm{C}_{8} \mathrm{H}_{13} \mathrm{O}_{3}(\mathrm{M}+\mathrm{H}): 157.0864 . \mathrm{Found}$ : 157.0863 .

exo-8a: mp decomposes; IR (KBr) 3395, 2920, $1717 \mathrm{~cm}^{-1}$; ${ }^{1} \mathrm{H}$ NMR (400 MHz, pyridine- $\left.d_{5}\right) \delta$ 4.58-4.54 (m, 1H) 2.74-2.66 (m, 4H) 2.58-2.51 (m, 1H) 2.27-2.21 (m, 2H) 1.99-1.93 (m, 1H) 1.74-1.70 (m, 1H); ${ }^{13} \mathrm{C}$ NMR (100 MHz, pyridine- $\left.d_{5}\right) \delta 213.1,70.1,65.2,52.5,52.3,46.7,34.1,17.5$; HRMS (FAB+) calcd. for $\mathrm{C}_{8} \mathrm{H}_{13} \mathrm{O}_{3}(\mathrm{M}+\mathrm{H})$ : 157.0864. Found: 157.0878 .

( \pm )-endo/exo-4-Benzyloxy-6-hydroxy-bicyclo[2.2.2]octan-2-one (8c). Ozone was bubbled through a solution of $6 \mathbf{c}(100 \mathrm{mg}, 0.410 \mathrm{mmol})$ in $\mathrm{CH}_{2} \mathrm{Cl}_{2}(8.5 \mathrm{~mL})$ at $-78{ }^{\circ} \mathrm{C}$ until it turned light blue (ca. $10 \mathrm{~min}$.). Excess ozone was then driven off by flushing with argon, where after DMS (160 $\mu \mathrm{L}$ ) was added and then the reaction mixture was allowed to reach room temperature. The solvent was removed at reduced pressure and to the resulting yellow oil was added heptane $(5 \mathrm{~mL})$, toluene $(2.5 \mathrm{~mL})$ and TEA $(2 \mathrm{~mL})$. Under intense stirring $\mathrm{SiO}_{2}\left(450 \mathrm{~m}^{2} / \mathrm{g}\right)(1.8 \mathrm{~g})$ was added and after a couple of minutes the colour had turned deep red. After completion of reaction (TLC) the silica gel was filtered off and washed with EtOAc. The filtrate was collected, the solvent removed at reduced pressure and the residue purified by column chromatography $\left(\mathrm{SiO}_{2}\right.$, Ether) to give endo-8c (51 mg, 50\%) and exo-8c (14 mg, 14\%) as white solids. 
endo-8c: $\mathrm{R}_{\mathrm{f}}($ ether $)=0.34 ; \mathrm{mp} 112.2-112.6^{\circ} \mathrm{C}$; IR $(\mathrm{KBr}) 3374,2938,1715 \mathrm{~cm}^{-1}$; ${ }^{1} \mathrm{H}$ NMR $\left(400 \mathrm{MHz}\right.$, benzene- $\left.d_{6}\right) \delta 7.24-7.10(\mathrm{~m}, 5 \mathrm{H}) 4.12$ $\left(\mathrm{d}_{\mathrm{AB}}, J_{\mathrm{AB}}=11.5 \mathrm{~Hz}, 1 \mathrm{H}\right) 4.08\left(\mathrm{~d}_{\mathrm{AB}}, J_{\mathrm{AB}}=11.5 \mathrm{~Hz}, 1 \mathrm{H}\right) 3.49-3.45(\mathrm{~m}, 1 \mathrm{H}) 2.52\left(\mathrm{dd}_{\mathrm{AB}}, J=2.2 \mathrm{~Hz}, J_{\mathrm{AB}}=17.8 \mathrm{~Hz}, 1 \mathrm{H}\right) 2.22\left(\mathrm{dd} \mathrm{AB}, J=3.3 \mathrm{~Hz}, J_{\mathrm{AB}}=\right.$ $17.8 \mathrm{~Hz}, 1 \mathrm{H}) 2.12-2.10(\mathrm{~m}, 1 \mathrm{H}) 1.81\left(\mathrm{qd}_{\mathrm{AB}}, J=3.3 \mathrm{~Hz}, J_{\mathrm{AB}}=13.5 \mathrm{~Hz}, 1 \mathrm{H}\right) 1.63\left(\mathrm{br} \mathrm{d}_{\mathrm{AB}}, J_{\mathrm{AB}}=13.5 \mathrm{~Hz}, 1 \mathrm{H}\right) 1.55(\mathrm{~s}, 1 \mathrm{H}) 1.28-1.21(\mathrm{~m}, 3 \mathrm{H}) 1.01-$ $0.97(\mathrm{~m}, 1 \mathrm{H}) ;{ }^{13} \mathrm{C}$ NMR $\left(100 \mathrm{MHz}\right.$, benzene- $\left.d_{6}\right) \delta 209.5,139.7,128.4,128.0,127.5,74.4,67.7,64.1,50.1,48.1,40.6,28.7,18.2 ; \mathrm{HRMS}(\mathrm{FAB}+)$ calcd. for $\mathrm{C}_{15} \mathrm{H}_{19} \mathrm{O}_{3}(\mathrm{M}+\mathrm{H})$ : 247.1334. Found: 247.1342. Anal. calcd. for $\mathrm{C}_{15} \mathrm{H}_{18} \mathrm{O}_{3}: \mathrm{C}, 73.15 ; \mathrm{H}, 7.37$. Found: $\mathrm{C}, 72.97 ; \mathrm{H}, 7.28$.

exo-8c: $\mathrm{R}_{\mathrm{f}}(\mathrm{ether})=0.48 ; \mathrm{mp} 87.8-89.2^{\circ} \mathrm{C}$; IR $(\mathrm{KBr}) 3460,2975,1710 \mathrm{~cm}^{-1} ;{ }^{1} \mathrm{H}$ NMR $\left(400 \mathrm{MHz}\right.$, benzene- $\left.d_{6}\right) \delta 7.25-7.11(\mathrm{~m}, 5 \mathrm{H}) 4.10(\mathrm{~s}, 2 \mathrm{H})$ 3.68-3.65 (m, 1H) 2.21-2.05 (m, 4H) $1.82\left(\mathrm{qd}_{\mathrm{AB}}, J=3.4 \mathrm{~Hz}, J_{\mathrm{AB}}=13.5 \mathrm{~Hz}, 1 \mathrm{H}\right) 1.70-1.68(\mathrm{~m}, 1 \mathrm{H}) 1.06\left(\operatorname{td}_{\mathrm{AB}}, J=3.3 \mathrm{~Hz}, J_{\mathrm{AB}}=13.5 \mathrm{~Hz}, 1 \mathrm{H}\right) 1.39-$ $1.38(\mathrm{~m}, 1 \mathrm{H}) 1.23-1.22(\mathrm{~m}, 1 \mathrm{H}) 1.08(\mathrm{br} \mathrm{s}, 1 \mathrm{H}) ;{ }^{13} \mathrm{C}$ NMR $\left(100 \mathrm{MHz}\right.$, benzene- $\left.d_{6}\right) \delta$ 209.5, 139.6, 128.5, 128.0, 127.5, 74.9, 64.77, 64.0, 50.9, 47.7, 41.5, 28.8, 16.1; HRMS (FAB+) calcd. for $\mathrm{C}_{15} \mathrm{H}_{19} \mathrm{O}_{3}(\mathrm{M}+\mathrm{H})$ : 247.1334. Found: 247.1337. Anal. calcd. for $\mathrm{C}_{15} \mathrm{H}_{18} \mathrm{O}_{3}$ : C, 73.15 ; $\mathrm{H}, 7.37$. Found: C, 73.06; H, 7.44 .

( \pm )-4-Hydroxy-bicyclo[2.2.2]oct-5-en-2-one (12). Maleic anhydride (9.18 g, $93.6 \mathrm{mmol}), 11$ (8.02 g, $71.5 \mathrm{mmol})$ and a few crystals of $p$-TsOH were added to isopropenyl acetate $(80 \mathrm{~mL}, 545 \mathrm{mmol})$ and the resulting mixture was refluxed for $12 \mathrm{~h}$, followed by removal of the solvent at reduced pressure. $\mathrm{H}_{2} \mathrm{O}(25 \mathrm{~mL})$ was added and the black oily residue was heated to $80{ }^{\circ} \mathrm{C}$. After $24 \mathrm{~h}$, the volatiles were removed at reduced pressure. Pyridine $(160 \mathrm{~mL}), \mathrm{H}_{2} \mathrm{O}(160 \mathrm{~mL})$ and TEA $(16 \mathrm{~mL})$ were added to the residue followed by $24 \mathrm{~h}$ of electrolysis according to Peterson et $a l .{ }^{3}$ Pyridine and $\mathrm{H}_{2} \mathrm{O}$ were removed at reduced pressure and the black residue was dissolved in $\mathrm{CH}_{2} \mathrm{Cl}_{2}$, filtrated through a pad of $\mathrm{SiO} \mathrm{O}_{2}$ and eluted with ether to give $12\left(5.43 \mathrm{~g}, 55 \%\right.$ ) as a yellow semi-crystalline oil. $\mathrm{R}_{\mathrm{f}}=0.19$ (heptane:EtOAc 1:1); IR (NaCl) $3408,2947,1720 \mathrm{~cm}^{-1} ;{ }^{1} \mathrm{H} \mathrm{NMR}$ $\left(400 \mathrm{MHz}\right.$, benzene- $\left.d_{6}\right) \delta 6.18(\mathrm{~d}, J=8.5 \mathrm{~Hz}, 1 \mathrm{H}) 5.64(\mathrm{dd}, J=8.5,6.4 \mathrm{~Hz}, 1 \mathrm{H}) 2.84-2.81(\mathrm{~m}, 1 \mathrm{H}) 2.15\left(\mathrm{dd} \mathrm{AB}, J=2.9 \mathrm{~Hz}, J_{\mathrm{AB}}=17.5 \mathrm{~Hz}, 1 \mathrm{H}\right) 1.99$

${ }^{3}$ Lightner, D. A.; Paquette, L. A.; Chayangkoon, P.; Lin, H. S.; Peterson, J. R.; Journal of Organic Chemistry 1988, 53, $1969-73$. 
$\left(\mathrm{d}_{\mathrm{AB}}, J_{\mathrm{AB}}=17.5 \mathrm{~Hz}, 1 \mathrm{H}\right) 1.43-1.28(\mathrm{~m}, 3 \mathrm{H}) 1.12-1.02(\mathrm{~m}, 1 \mathrm{H}) ;{ }^{13} \mathrm{C}$ NMR $\left(100 \mathrm{MHz}\right.$, benzene- $\left.d_{6}\right) \delta 207.9,141.8,125.5,72.2,48.4,48.0,32.8$, 21.6; HRMS (FAB+) calcd. for $\mathrm{C}_{8} \mathrm{H}_{11} \mathrm{O}_{2}(\mathrm{M}+\mathrm{H})$ : 139.0759. Found: 139.0750. Anal. calcd for $\mathrm{C}_{8} \mathrm{H}_{10} \mathrm{O}_{2}: \mathrm{C}, 69.54$; H, 7.30. Found: C, 69.66; $\mathrm{H}$, 7.25.

( \pm )-tert-Butyl-(5,5-dimethoxy-bicyclo[2.2.2]oct-2-en-1-yloxy)-dimethyl-silane (14). Trimethyl orthoformate $(0.48 \mathrm{~mL}, 4.4 \mathrm{mmol}), 12$ (403 $\mathrm{mg}, 2.9 \mathrm{mmol})$, and a few crystals of $p-\mathrm{TsOH}$ were added to methanol $(5 \mathrm{~mL})$ and stirred at $\mathrm{rt}$ over night. Saturated aqueous $\mathrm{NaHCO}_{3} \mathrm{was}$ then added and the mixture was extracted with EtOAc $(4 \times 8 \mathrm{~mL})$. The organic phase was washed with brine $(15 \mathrm{~mL})$ and dried $\left(\mathrm{Na}_{2} \mathrm{SO}_{4}\right)$ before removal of the solvent under reduced pressure giving crude $\mathbf{1 3}$ as yellow crystals. NaH (60\% in mineral oil, $524 \mathrm{mg}, 13.1 \mathrm{mmol})$ was washed three times with pentane under argon atmosphere before addition of some crystals of 18 -crown-6 dissolved in THF ( $4 \mathrm{~mL})$. The mixture was cooled to $0{ }^{\circ} \mathrm{C}$ before addition of crude $\mathbf{1 3}$ in THF ( $3 \mathrm{~mL})$. After 20 minutes, TBDMSCl (1.97 g, $13.1 \mathrm{mmol})$ in THF $(5 \mathrm{~mL})$ was added and the mixture was stirred at $\mathrm{rt}$ for $12 \mathrm{~h}, \mathrm{H}_{2} \mathrm{O}$ was added and the mixture was extracted with EtOAc $(3 \times 8 \mathrm{~mL})$. The organic phase was then dried $\left(\mathrm{Na}_{2} \mathrm{SO}_{4}\right)$ before removal of the solvent at reduced pressure. The residue was purified by column chromatography $\left(\mathrm{SiO}_{2}\right.$, heptane:EtOAc, $\left.23: 2\right)$ to give 14 (451 mg, $52 \%$ over two steps) as a clear oil. $\mathrm{R}_{\mathrm{f}}=0.30$ (heptane:EtOAc $\left.23: 2\right) ;{ }^{1} \mathrm{H}$ NMR $\left(400 \mathrm{MHz}\right.$, benzene- $\left.d_{6}\right) \delta 6.29(\mathrm{~d}, J=8.5 \mathrm{~Hz}, 1 \mathrm{H}) 6.01(\mathrm{dd}, J=6.7$, $8.5 \mathrm{~Hz}, 1 \mathrm{H}) 3.01(\mathrm{~s}, 3 \mathrm{H}) 2.97(\mathrm{~s}, 3 \mathrm{H}) 2.63-2.60(\mathrm{~m}, 1 \mathrm{H}) 1.99\left(\mathrm{dd}_{\mathrm{AB}}, J=3.4 \mathrm{~Hz}, J_{\mathrm{AB}}=12.1 \mathrm{~Hz}, 1 \mathrm{H}\right) 1.88\left(\mathrm{~d}_{\mathrm{AB}}, J_{\mathrm{AB}}=12.1 \mathrm{~Hz}, 1 \mathrm{H}\right) 1.92-1.85(\mathrm{~m}$, 1H) $1.76(\mathrm{dt}, J=4.4,10.5 \mathrm{~Hz}, 1 \mathrm{H}) 1.53-1.46(\mathrm{~m}, 1 \mathrm{H}) 1.16-1.06(\mathrm{~m}, 1 \mathrm{H}) 1.01(\mathrm{~s}, 9 \mathrm{H}) 0.14(\mathrm{~s}, 6 \mathrm{H}) ;{ }^{13} \mathrm{C}$ NMR $\left(100 \mathrm{MHz}\right.$, benzene- $\left.d_{6}\right) \delta 139.1$, 129.1, 105.8, 76.0, 48.2, 47.9, 47.5, 36.9, 33.5, 26.0, 21.2, 18.2, -2.0 (2C); HRMS (EI+) calcd. for $\mathrm{C}_{16} \mathrm{H}_{30} \mathrm{O}_{3} \mathrm{Si}$ (M): 298.1964. Found: 298.1966.

( \pm )-exo-1-(tert-Butyldimethylsilanyloxy)-5,5-dimethoxy-bicyclo[2.2.2]octan-2-ol (16). At $0{ }^{\circ} \mathrm{C}, 1 \mathrm{M} \mathrm{BH}_{3} \times \mathrm{THF}(0.54 \mathrm{~mL}, 0.54 \mathrm{mmol})$ was added drop wise to a solution of $\mathbf{1 4}(83 \mathrm{mg}, 0.28 \mathrm{mmol})$ in THF $(4 \mathrm{~mL})$. The reaction mixture was allowed to reach $\mathrm{rt}$ and stirred for $4 \mathrm{~h}$ before careful addition of $\mathrm{H}_{2} \mathrm{O}(2 \mathrm{~mL})$ followed by $2 \mathrm{M} \mathrm{NaOH}(0.85 \mathrm{~mL}, 1.7 \mathrm{mmol})$ and $30 \% \mathrm{H}_{2} \mathrm{O}_{2}(0.12 \mathrm{~mL}, 1.2 \mathrm{mmol})$. After $12 \mathrm{~h}$, the aqueous phase 
was saturated with $\mathrm{NaCl}(\mathrm{s})$ before extraction with EtOAc $(3 \times 5 \mathrm{~mL})$. The organic phase was washed with brine $(10 \mathrm{~mL})$ and dried $\left(\mathrm{Na}_{2} \mathrm{SO}_{4}\right)$ before removal of the solvent at reduced pressure. The residue was purified by column chromatography $\left(\mathrm{SiO}_{2}\right.$, heptane:EtOAc 95:5) to give exo-16 (29 $\mathrm{mg}, 33 \%$ ) as yellow crystals. $\mathrm{R}_{\mathrm{f}}=0.18$ (heptane:EtOAc 23:2); IR (KBr) 3583, $2936 \mathrm{~cm}^{-1} ;{ }^{1} \mathrm{H}$ NMR (400 MHz, benzene- $\left.d_{6}\right) \delta 4.77-4.74(\mathrm{~m}, 1 \mathrm{H})$ $3.73(\mathrm{~s}, 6 \mathrm{H}) 2.82-2.77(\mathrm{~m}, 1 \mathrm{H}) 2.57-2.49(\mathrm{~m}, 2 \mathrm{H})$ 2.39-2.29 (m, 3H) 2.22-2.20 (m, 1H) 1.89-1.82 (m, 2H) $1.60(\mathrm{dt}, J=3.1,13.7 \mathrm{~Hz}, 1 \mathrm{H}) 1.16(\mathrm{~s}$, 9H) $0.04(\mathrm{~s}, 6 \mathrm{H}) ;{ }^{13} \mathrm{C}$ NMR $\left(100 \mathrm{MHz}\right.$, benzene- $\left.d_{6}\right) \delta 104.2,73.5,72.0,48.1,47.9,43.8,34.8,32.2,26.7,26.2,22.3,18.4,-4.1,-4.6$; HRMS (FAB+) calcd. for $\mathrm{C}_{16} \mathrm{H}_{33} \mathrm{O}_{4} \mathrm{Si}(\mathrm{M}+\mathrm{H}): 317.2150$. Found: 317.2138 .

( \pm )-4-(tert-Butyldimethylsilanyloxy)-bicyclo[2.2.2]oct-5-en-2-one (17). At -10 ${ }^{\circ} \mathrm{C}$, TBDMSOTf $(13 \mathrm{~mL}, 57 \mathrm{mmol})$ was added drop wise to a solution of 2,6-lutidine $(11.0 \mathrm{~mL}, 94.0 \mathrm{mmol})$ and $\mathbf{1 2}(5 \mathrm{~g}, 36.2 \mathrm{mmol})$ in $\mathrm{CH}_{2} \mathrm{Cl}_{2}(40 \mathrm{~mL})$. The mixture was stirred for $2 \mathrm{~h}$ at $0{ }^{\circ} \mathrm{C}$, followed by addition of $\mathrm{H}_{2} \mathrm{O}(15 \mathrm{~mL})$ and extraction with $\mathrm{CH}_{2} \mathrm{Cl}_{2}(4 \times 10 \mathrm{~mL})$. The organic phase was washed with brine (30 $\left.\mathrm{mL}\right)$ before removal of the solvent at reduced pressure. The crude product was dissolved in THF $(20 \mathrm{~mL})$ before addition of $1 \mathrm{M} \mathrm{HCl}(8 \mathrm{~mL})$. After $2 \mathrm{~h}$ of stirring at rt, saturated aqueous $\mathrm{NaHCO}_{3}$ was added and the mixture was extracted with EtOAc $(4 \times 15 \mathrm{~mL})$. The organic phase was then washed with brine $(30 \mathrm{~mL})$ and dried $\left(\mathrm{Na}_{2} \mathrm{SO}_{4}\right)$ before removal of solvent at reduced pressure. The residue was distilled (bp $\left.84{ }^{\circ} \mathrm{C} / 0.15 \mathrm{mbar}\right)$ to give $\mathbf{1 7}(6.4 \mathrm{~g}, 70 \%)$ as a clear oil. $\mathrm{R}_{\mathrm{f}}=0.30$ (heptane:EtOAc 87:13); IR (NaCl) 2954, $1735 \mathrm{~cm}^{-1} ;{ }^{1} \mathrm{H}$ NMR $\left(400 \mathrm{MHz}\right.$, benzene- $\left.d_{6}\right) \delta 6.17(\mathrm{~d}, J=8.6 \mathrm{~Hz}, 1 \mathrm{H}) 5.64(\mathrm{dd}, J=8.4$, $6.7 \mathrm{~Hz}, 1 \mathrm{H}) 2.85-2.84(\mathrm{~m}, 1 \mathrm{H}) 2.21\left(\mathrm{dd}_{\mathrm{AB}}, J=3.1 \mathrm{~Hz}, J_{\mathrm{AB}}=17.4 \mathrm{~Hz}, 1 \mathrm{H}\right) 2.05\left(\mathrm{~d}_{\mathrm{AB}}, J_{\mathrm{AB}}=17.3 \mathrm{~Hz}, 1 \mathrm{H}\right) 1.49-1.42(\mathrm{~m}, 1 \mathrm{H}) 1.38-1.29(\mathrm{~m}, 2 \mathrm{H})$ $1.11-1.03(\mathrm{~m}, 1 \mathrm{H}) 0.93(\mathrm{~s}, 9 \mathrm{H})-0.01(\mathrm{~s}, 6 \mathrm{H}) ;{ }^{13} \mathrm{C}$ NMR $\left(100 \mathrm{MHz}\right.$, benzene- $\left.d_{6}\right) \delta 206.3,142.3,125.4,75.7,48.6,48.4,33.7,26.0,21.7,18.2,-2.2$ (2C); HRMS (FAB+) calcd. for $\mathrm{C}_{14} \mathrm{H}_{23} \mathrm{O}_{3} \mathrm{Si}(\mathrm{M}-\mathrm{H})$ : 251.1468. Found: 251.1467. Anal calcd for $\mathrm{C}_{14} \mathrm{H}_{24} \mathrm{O}_{\mathrm{s}} \mathrm{Si}$ : C, 66.61; H, 9.58. Found: C, 66.51; H, 9.66 .

( \pm )-4-(tert-Butyl-dimethyl-silanyloxy)-bicyclo[2.2.2]octane-2,6-diol (18) and ( \pm )-1-(tert-Butyl-dimethyl-silanyloxy)-bicyclo[2.2.2]octane2,5-diol (19) as diastereomeric mixtures. At $0{ }^{\circ} \mathrm{C}, 1 \mathrm{M} \mathrm{BH} \times 3 \mathrm{THF}(1.6 \mathrm{~mL}, 1.6 \mathrm{mmol})$ was added drop wise to a solution of $12(100 \mathrm{mg}$, 0.4 
mmol) in THF ( $4 \mathrm{~mL})$. The reaction mixture was then allowed to reach $\mathrm{rt}$ and stirred for $3 \mathrm{~h}$ before careful addition of $\mathrm{H}_{2} \mathrm{O}(2 \mathrm{~mL})$ followed by 2 $\mathrm{M} \mathrm{NaOH}(1.2 \mathrm{~mL}, 2.4 \mathrm{mmol})$ and $30 \% \mathrm{H}_{2} \mathrm{O}_{2}(0.25 \mathrm{~mL}, 2.4 \mathrm{mmol})$. After $12 \mathrm{~h}$, the aqueous phase was saturated with $\mathrm{NaCl}(\mathrm{s})$ followed by extraction with EtOAc $(4 \times 5 \mathrm{~mL})$. The organic phase was washed with brine $(15 \mathrm{~mL})$ and dried $\left(\mathrm{Na}_{2} \mathrm{SO}_{4}\right)$ before removal of solvent at reduced pressure. The residue was purified by column chromatography $\left(\mathrm{SiO}_{2}, \mathrm{CH}_{2} \mathrm{Cl}_{2}\right.$ :ether 1:1) to give diastereomeric mixtures of $\mathbf{1 8}(43 \mathrm{mg}, 40 \%)$ and 19 (45 mg, $42 \%)$ as white crystals in a ratio of 49:51, respectively.

18: $\mathrm{R}_{\mathrm{f}}=0.15$ (heptane:EtOAc 1:1); IR (KBr) 3354, $2929 \mathrm{~cm}^{-1} ;{ }^{1} \mathrm{H}$ NMR (400 MHz, benzene- $\left.d_{6}\right) \delta$ 4.25-4.23 (m, 1H) 3.62-3.57 (m, $\left.1 \mathrm{H}\right) 2.10-1.97$ $(\mathrm{m}, 2 \mathrm{H}) 1.85-1.79(\mathrm{~m}, 1 \mathrm{H}) 1.58-1.50(\mathrm{~m}, 1 \mathrm{H}) 1.44-1.23(\mathrm{~m}, 5 \mathrm{H}) 1.09-1.01(\mathrm{~m}, 1 \mathrm{H})$ 0.99-0.97 (m, 9H+1H) $0.07(\mathrm{~d}, J=3.2 \mathrm{~Hz}, 6 \mathrm{H}) ;{ }^{13} \mathrm{C} \mathrm{NMR}(100$ $\mathrm{MHz}$, benzene- $\left.d_{6}\right) \delta 72.4,68.7,65.4,46.4,45.4,39.5,33.2,26.1,18.2,17.9,1.4,-1.7$; HRMS (FAB+) calcd. for $\mathrm{C}_{14} \mathrm{H}_{29} \mathrm{O}_{3} \mathrm{Si}(\mathrm{M}+\mathrm{H}): 273.1886$. Found: 273.1876.

19: $\mathrm{R}_{\mathrm{f}}=0.22$ (heptane:EtOAc 1:1); IR (KBr) 3345, $2929 \mathrm{~cm}^{-1} ;{ }^{1} \mathrm{H}$ NMR (400 MHz, benzene- $\left.d_{6}\right) \delta 3.94(\mathrm{dq}, J=1.6,9.3 \mathrm{~Hz}, 1 \mathrm{H}) 3.61-3.57(\mathrm{~m}$, 1H) $2.45-2.38(\mathrm{~m}, 1 \mathrm{H}) 2.05(\mathrm{dd}, J=9.3,13.7 \mathrm{~Hz}, 1 \mathrm{H}) 1.98-1.91(\mathrm{~m}, 1 \mathrm{H}) 1.57-1.50(\mathrm{~m}, 2 \mathrm{H}) 1.37(\mathrm{dt}, J=3.1,13.7 \mathrm{~Hz}) 1.31-1.12(\mathrm{~m}, 5 \mathrm{H}) 0.92(\mathrm{~s}$, 9H) $0.03(\mathrm{~s}, 6 \mathrm{H}) ;{ }^{13} \mathrm{C}$ NMR $\left(100 \mathrm{MHz}\right.$, benzene- $\left.d_{6}\right) \delta 73.5,71.0,69.7,42.6,33.3,32.9,26.1,26.0,23.7,18.2,-4.2,-4.8$; HRMS (FAB+) calcd. for $\mathrm{C}_{14} \mathrm{H}_{29} \mathrm{O}_{3} \mathrm{Si}(\mathrm{M}+\mathrm{H}): 273.1886$. Found: 273.1874 .

\section{4-(tert-Butyldimethylsilanyloxy)-bicyclo[2.2.2]octan-2,6-dione (3).}

c) Jones' oxidation of 18: At $0{ }^{\circ} \mathrm{C}$, a chromic acid solution $\left(\mathrm{CrO}_{3}(1.05 \mathrm{mg}, 1.05 \mathrm{mmol}) /\right.$ conc. $\left.\mathrm{H}_{2} \mathrm{SO}_{4}(2.5 \mathrm{~mL}) / \mathrm{H} 2 \mathrm{O}(4 \mathrm{~mL})\right)$ was added drop wise to a solution of $\mathbf{1 8}(100 \mathrm{mg}, 0.37 \mathrm{mmol})$ in acetone $(6 \mathrm{~mL})$. The reaction mixture was then allowed to reach $\mathrm{rt}$ and stirred for $2 \mathrm{~h}$ before addition of saturated aqueous $\mathrm{NaHCO}_{3}$. The mixture was extracted with EtOAc $(4 \times 5 \mathrm{~mL})$ and the organic phase was dried $\left(\mathrm{Na}_{2} \mathrm{SO}_{4}\right)$ before removal of the solvent at reduced pressure. The residue was filtrated through a pad of $\mathrm{SiO}_{2}$ and eluted with heptane:EtOAc (8:2) to give 3 (63 mg, $64 \%$ ) as white crystals. 
4-Hydroxy-bicyclo[2.2.2] octane-2,6-dione (2). To a solution of 3 (225 mg, $0.84 \mathrm{mmol})$ in dry $\mathrm{MeCN}(5.0 \mathrm{~mL}) \mathrm{was} \operatorname{added~} \mathrm{BF}_{3} \cdot \mathrm{Et}_{2} \mathrm{O}(170 \mu \mathrm{L}$, $1.3 \mathrm{mmol}$ ) drop wise at $-10{ }^{\circ} \mathrm{C}$. The resulting mixture was stirred for 40 minutes where after $0.4 \mathrm{M} \mathrm{NaHCO}_{3}$ aqueous solution $(5.0 \mathrm{~mL})$ and EtOAc $(5.0 \mathrm{~mL})$ were added and stirred for an additional five minutes at $-10{ }^{\circ} \mathrm{C}$. The aqueous phase was then extracted with EtOAc $(5 \times 5 \mathrm{~mL})$ and the combined organic phases were washed with brine and dried $\left(\mathrm{Na}_{2} \mathrm{SO}_{4}\right)$. After concentration at reduced pressure the residue was purified by column chromatography $\left(\mathrm{SiO}_{2}\right.$, EtOAc:toluene 65:35) to give $2(64 \mathrm{mg}, 50 \%)$ as a white powder. $\mathrm{mp}$ decomposes; $\mathrm{IR}(\mathrm{KBr}) 3364,2939,2920,1717 \mathrm{~cm}^{-1}$; ${ }^{1} \mathrm{H}$ NMR $\left(400 \mathrm{MHz}\right.$, benzene- $\left.d_{6}\right) \delta 2.99-2.98(\mathrm{~m}, 1 \mathrm{H}) 1.99\left(\mathrm{br} \mathrm{dd}_{\mathrm{AB}}, J_{\mathrm{AB}}=18.1 \mathrm{~Hz}, 2 \mathrm{H}\right) 1.86\left(\right.$ br dd $\left.\mathrm{AB}, J_{\mathrm{AB}}=18.2 \mathrm{~Hz}, 2 \mathrm{H}\right) 1.16-1.06(\mathrm{~m}, 4 \mathrm{H}) 0.69$ $(\mathrm{s}, 1 \mathrm{H}) ;{ }^{13} \mathrm{C}$ NMR $\left(100 \mathrm{MHz}\right.$, benzene- $\left.d_{6}\right) \delta$ 202.6, 69.6, 63.4, 51.7, 32.1, 20.2; HRMS (FAB+) calcd. for $\mathrm{C}_{8} \mathrm{H}_{11} \mathrm{O}_{3}(\mathrm{M}+\mathrm{H}): 155.0709 . \mathrm{Found}$ : 155.0712. Anal. calcd for $\mathrm{C}_{8} \mathrm{H}_{10} \mathrm{O}_{3}$ : C, 62.33; H, 6.54. Found: $\mathrm{C}, 62.08 ; \mathrm{H}, 6.60$. 
Table 1. Synthesis of $\mathbf{3}$ via hydroboration-oxidation.

\begin{tabular}{|c|c|c|c|c|c|}
\hline Entry & Borane & Ox. Reagent & Solvent & Scale (mmol) & Yield (\%) \\
\hline 1 & $\mathrm{BH}_{3} * \mathrm{THF}$ & $\mathrm{PCC} / \mathrm{MS} 4 \AA^{a}$ & $\mathrm{CH}_{2} \mathrm{Cl}_{2}$ & 0.8 & Trace $^{c}$ \\
\hline 2 & $\mathrm{BH}_{3} * \mathrm{THF}$ & $\mathrm{PCC} / \mathrm{MS} 4 \AA^{b}$ & $\mathrm{CH}_{2} \mathrm{Cl}_{2}$ & 0.8 & 35 \\
\hline 3 & $\mathrm{BH}_{3} * \mathrm{SMe}_{2}$ & NMO/TPAP/MS $4 \AA^{a}$ & Ether & 0.5 & 14 \\
\hline 4 & $\mathrm{BH}_{3} * \mathrm{SMe}_{2}$ & NMO/TPAP/MS 4A $\AA^{b}$ & Ether & 1.0 & 42 \\
\hline 5 & $\mathrm{BH}_{3} * \mathrm{SMe}_{2}$ & NMO/TPAP/MS $4 \AA^{b}$ & Ether & 2.0 & 40 \\
\hline 6 & $\mathrm{BH}_{3}{ }^{*} \mathrm{THF}$ & NMO/TPAP/MS $4 \AA^{b}$ & Ether & 1.0 & 40 \\
\hline 7 & $\mathrm{BH}_{3} * \mathrm{THF}$ & $\mathrm{KMnO}_{4} \mathrm{CuSO}_{4} * 5 \mathrm{H}_{2} \mathrm{O}$ & Benzene & 0.5 & - \\
\hline 8 & $\mathrm{BH}_{3} * \mathrm{SMe}_{2}$ & $\mathrm{FeBr}_{2} / \mathrm{H}_{2} \mathrm{O}_{2}$ & $\mathrm{CH}_{3} \mathrm{CN}$ & 0.5 & - \\
\hline
\end{tabular}

${ }^{a}$ One-step procedure; ${ }^{b}$ Two-step procedure via isolation of the organo borane followed by oxidation; ${ }^{c}$ As indicated by TLC 
As can be seen from Table 1 two additional methods for the oxidation of secondary alcohols were tested in the hydroboration-oxidation sequence; $\mathrm{KMnO}_{4} / \mathrm{CuSO}_{4} \cdot 5 \mathrm{H}_{2} \mathrm{O}$ in benzene ${ }^{4}$ and $\mathrm{FeBr}_{3} / \mathrm{H}_{2} \mathrm{O}_{2}$ in $\mathrm{CH}_{3} \mathrm{CN}$. ${ }^{5}$ However, formation of diketone 3 could not be detected.

\footnotetext{
${ }^{4}$ Paquette, L. A.; Tsui, H.-C. Journal of Organic Chemistry 1996, 61, 142-5.

${ }^{5}$ Martin, S. E.; Garrone, A. Tetrahedron Letters 2002, 44, 549-52.
} 
${ }^{1}$ H NMR spectrum of compound 9
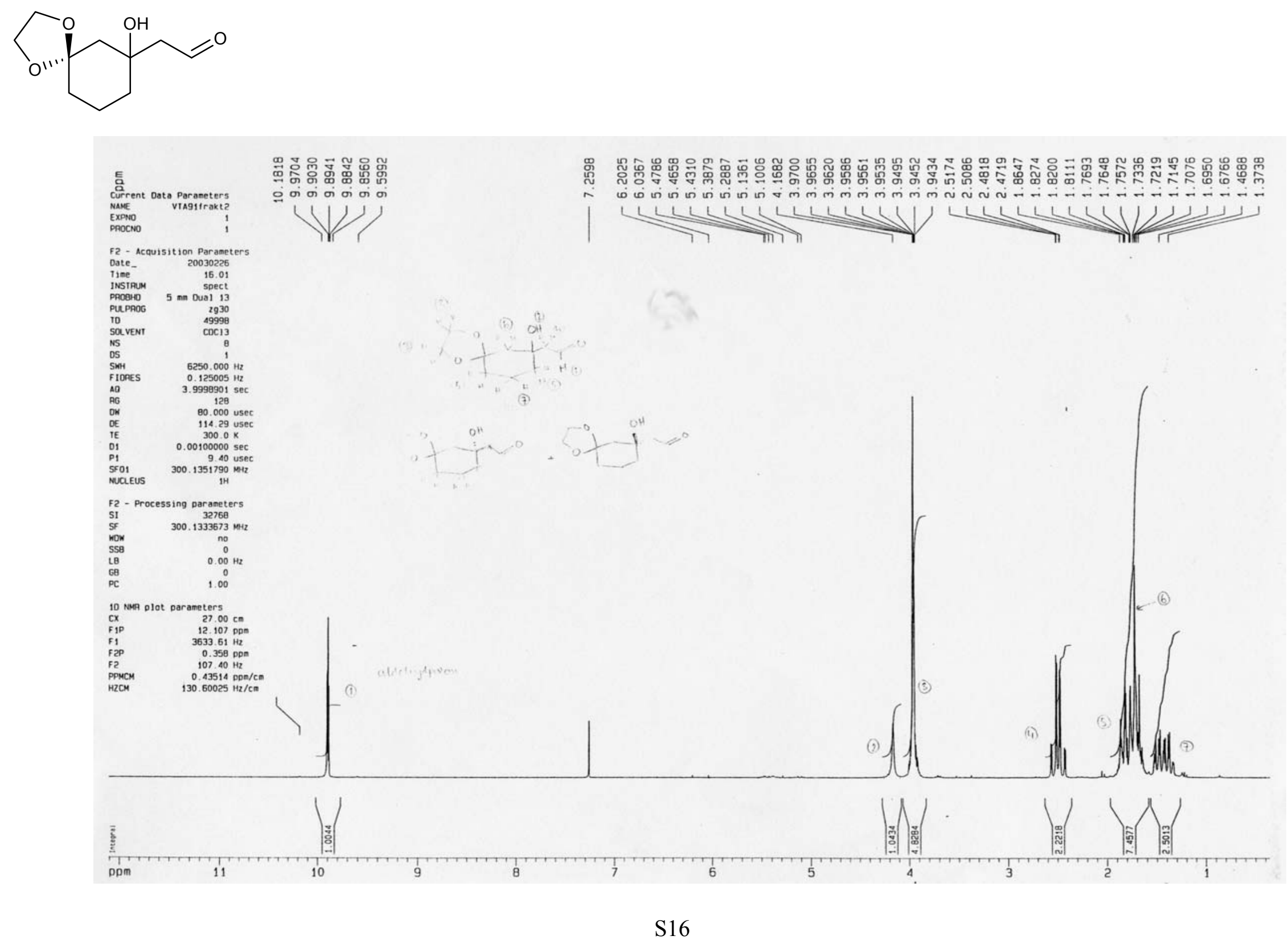
${ }^{13} \mathrm{C}$ NMR spectrum of compound 9

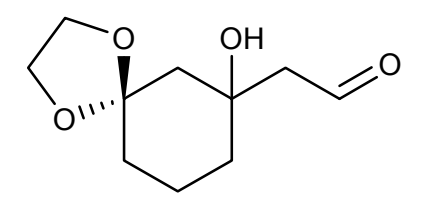

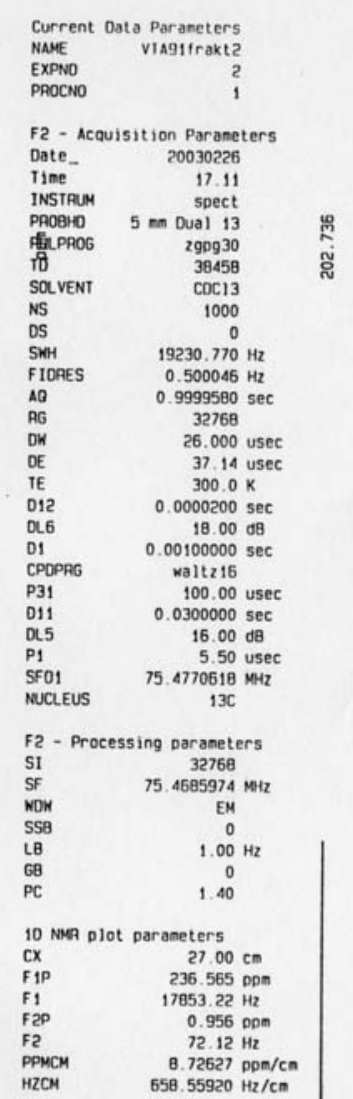

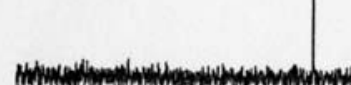

ppm' ' $2200^{\prime}$ ' ' '

180

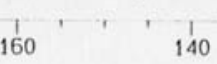

140

120

100

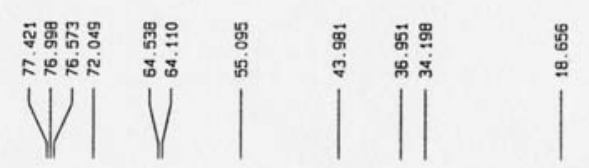


${ }^{1} \mathrm{H}$ NMR spectrum of compound $\mathbf{1 0}$
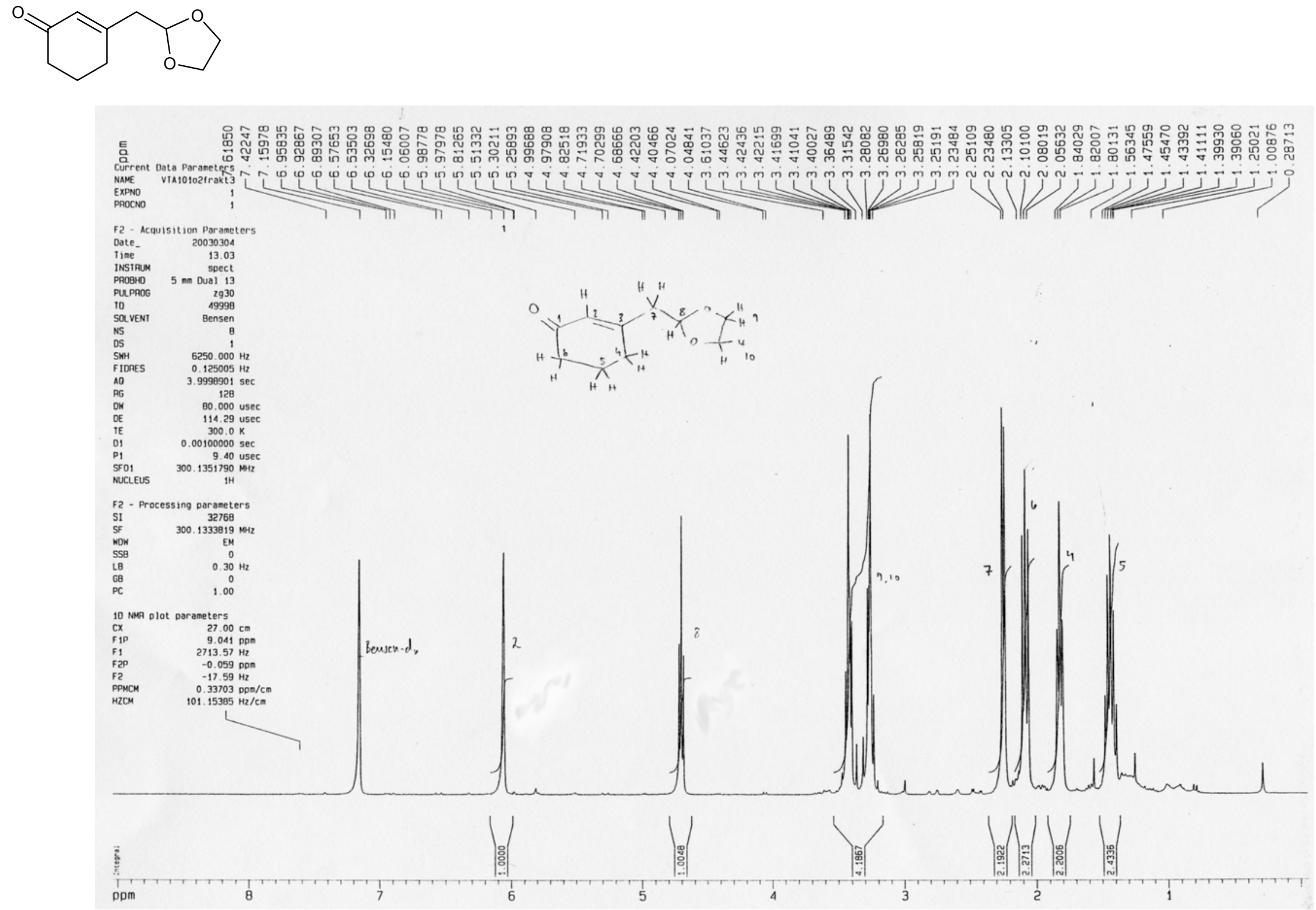
${ }^{13} \mathrm{C}$ NMR spectrum of compound $\mathbf{1 0}$<smiles>O=C1C=C(CC2OCCO2)CCC1</smiles>

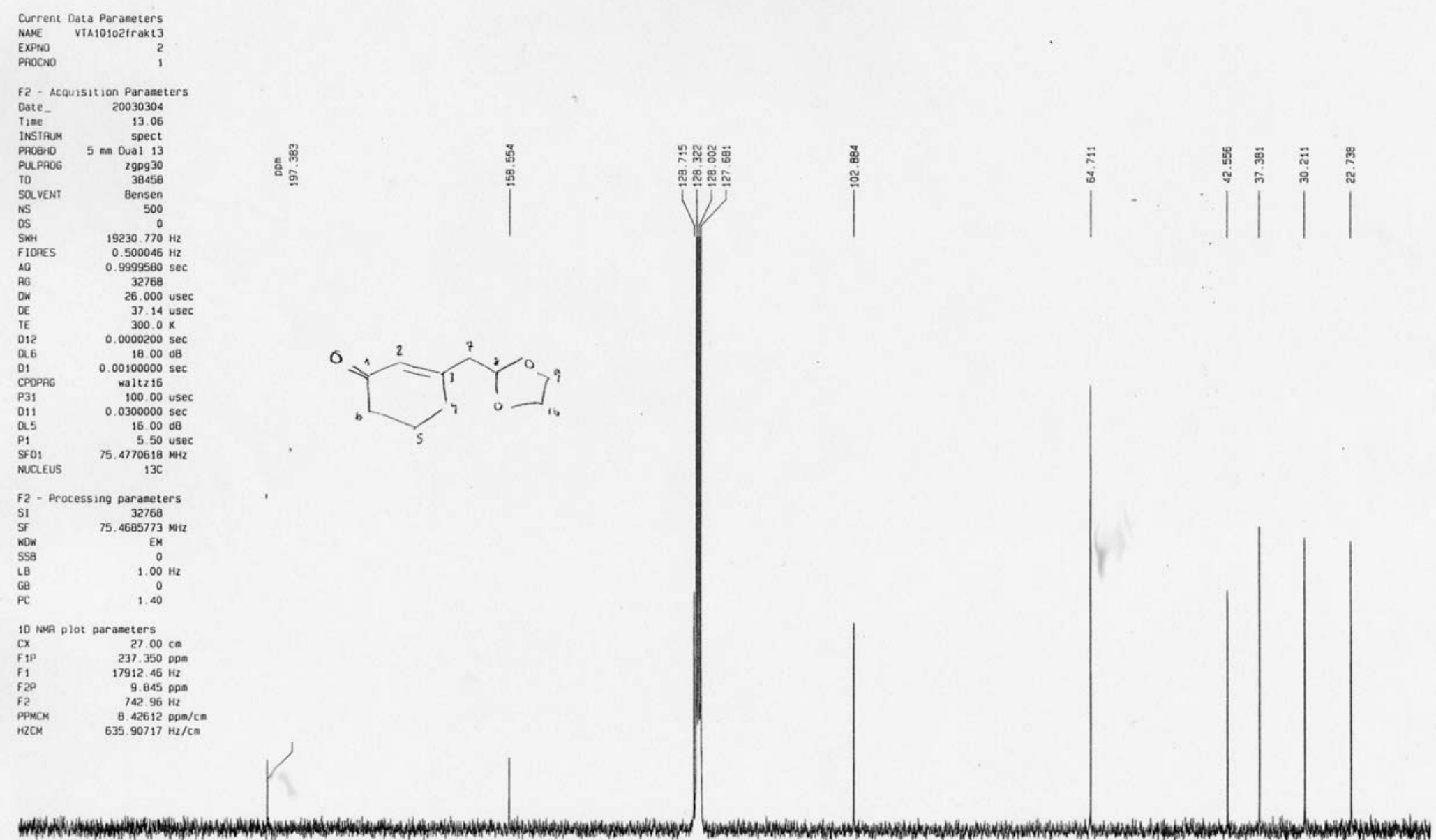

ppm $220 \quad 200 \quad 180 \quad 160 \quad 140$

120

100

80

60

40

20 
${ }^{1} \mathrm{H}$ NMR spectrum of compound $\mathbf{1 4}$
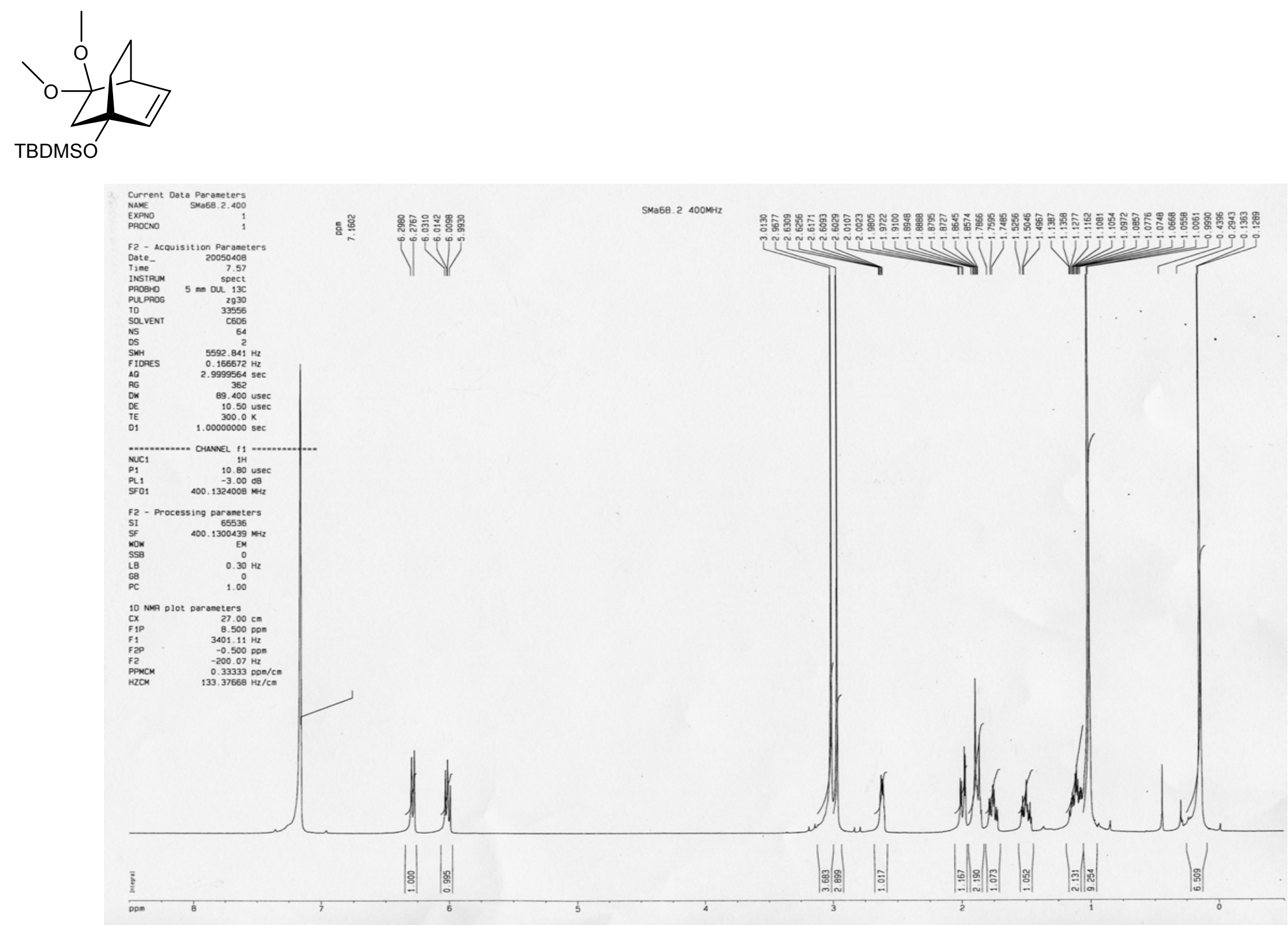
${ }^{13} \mathrm{C}$ NMR spectrum of compound $\mathbf{1 4}$

TBDMSO

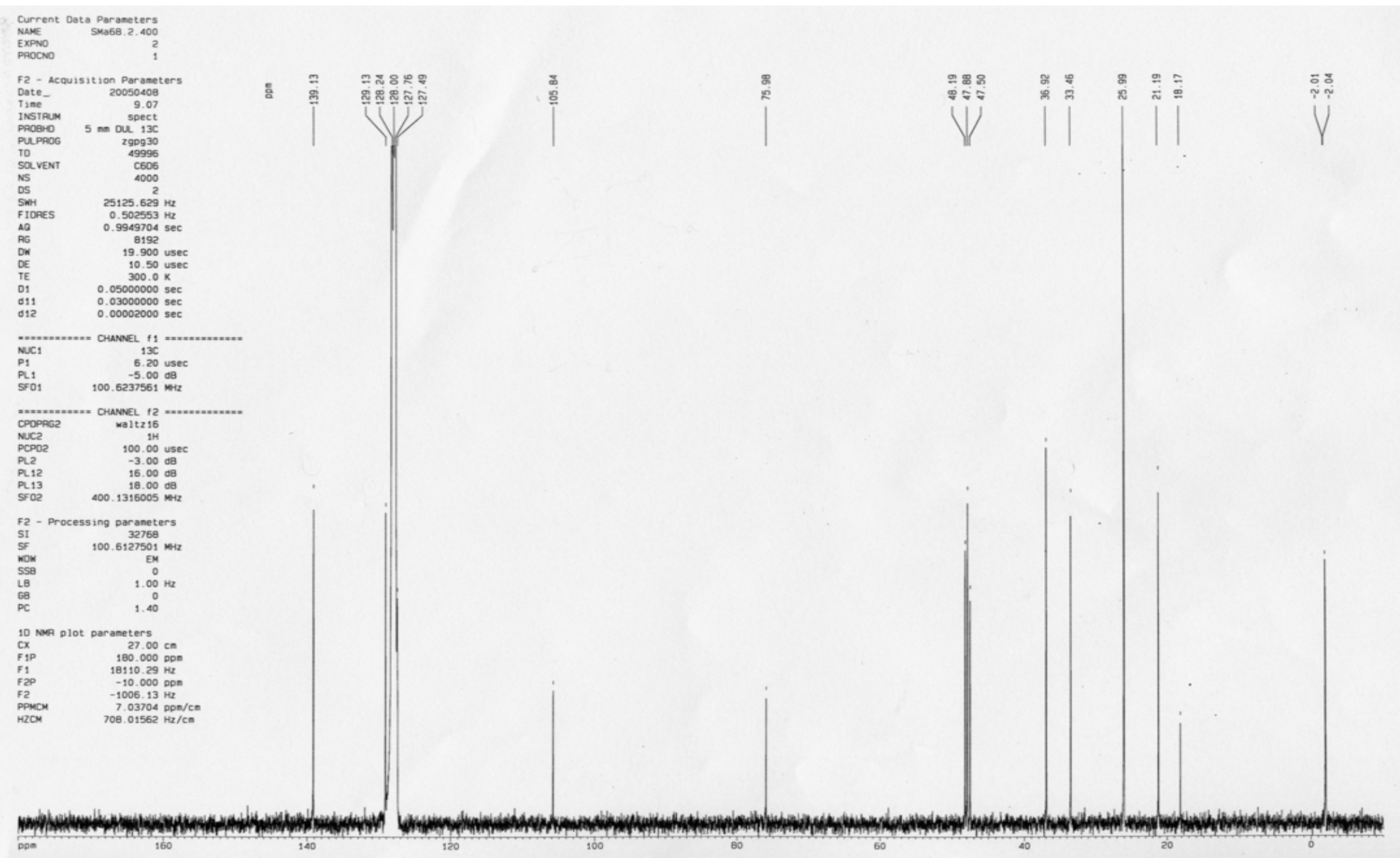


${ }^{1}$ H NMR spectrum of compound $\mathbf{1 6}$
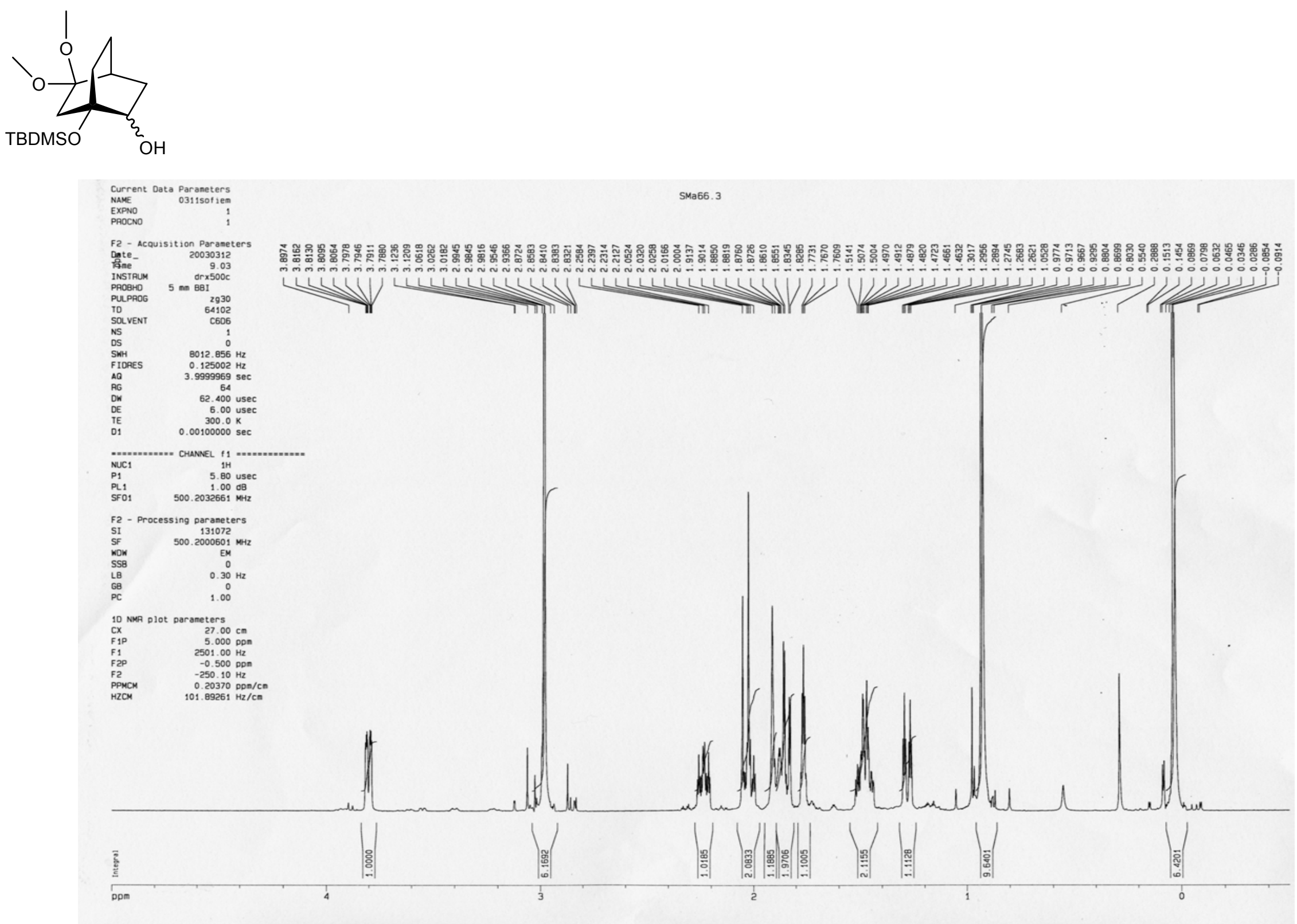
${ }^{13} \mathrm{C}$ NMR spectrum of compound $\mathbf{1 6}$
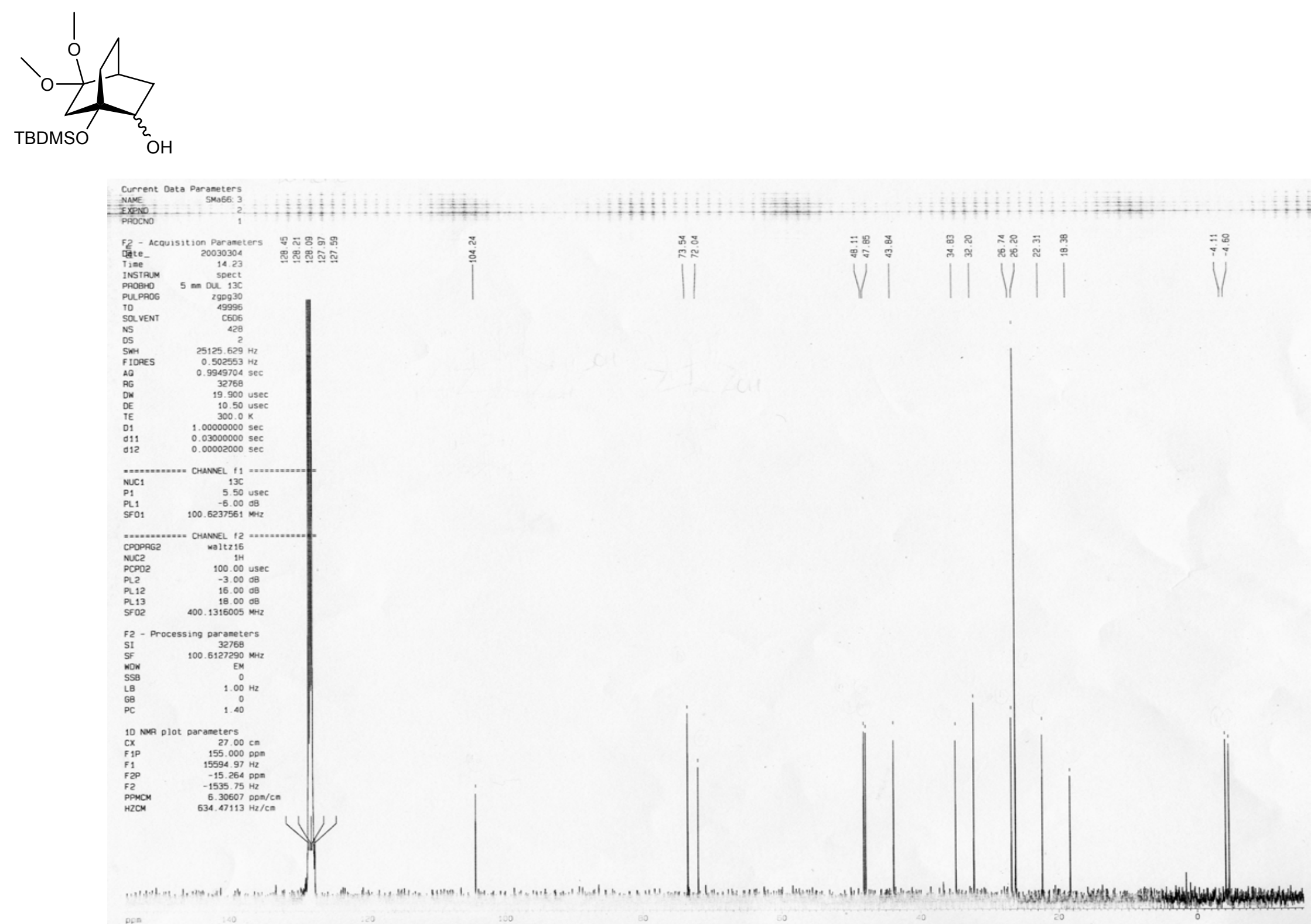